\title{
DESIGN OF THE BEAM DELIVERY SYSTEM FOR THE INTERNATIONAL LINEAR COLLIDER
}

A.Seryi, J.Amann, R.Arnold, F.Asiri, K.Bane, P.Bellomo, E.Doyle, A.Fasso, K.Jonghoon, L.Keller, K.Ko, Z.Li, T.Markiewicz, T.Maruyama, K.Moffeit, S.Molloy, Y.Nosochkov, N.Phinney, T.Raubenheimer, S.Seletskiy, S.Smith, C.Spencer, P.Tenenbaum, D.Walz, G.White, M.Woodley, M.Woods, L.Xiao (SLAC),M.Anerella, A.Jain, A.Marone, B.Parker (BNL),O.Delferriere, O.Napoly, J.Payet, D.Uriot (CEA), N.Watson (Birmingham Univ.), I.Agapov, J-L.Baldy, D.Schulte (CERN), G.Burt, A.Dexter (Lancaster Univ.), K.Buesser, W.Lohmann (DESY), L.Bellantoni, A.Drozhdin, V.Kashikhin, V.Kuchler, T.Lackowski, N.Mokhov, N.Nakao, T.Peterson, M.Ross, S.Striganov, J.Tompkins, M.Wendt, X.Yang (FNAL), A.Enomoto, S.Kuroda, T.Okugi, T.Sanami, Y.Suetsugu, T.Tauchi (KEK), M.del Carmen Alabau, P.Bambade, J.Brossard, O.Dadoun (LAL), P.Burrows, G.Christian, C.Clarke, B.Constance, H.Dabiri Khah, A.Hartin, C.Perry, C.Swinson (Oxford), A.Ferrari (Uppsala Univ.), G.Blair, S.Boogert, J.Carter (RHUL), D. Angal-Kalinin, C.Beard, C.Densham, L.Fernandez-Hernando, J.Greenhalgh, P.Goudket, F.Jackson, J.Jones, A.Kalinin, L. Ma, P. McIntosh (STFC), H. Yamamoto (Tohoku Univ.), T.Mattison (UBC, Vancouver), J.Carwardine, C.Saunders (ANL), R.Appleby (Manchester Univ.), E.Torrence (Univ. Oregon), J.Gronberg (LLNL), T.Sanuki (Univ. Tokyo), Y.Iwashita (Kyoto Univ.), V.Telnov (BINP), D.Warner (Univ. Colorado)

\section{Abstract}

The beam delivery system for the linear collider focuses beams to nanometer sizes at its interaction point, collimates the beam halo to provide acceptable background in the detector and has a provision for state-of-the art beam instrumentation in order to reach the ILCs physics goals. This paper describes the design details and status of the baseline configuration considered for the reference design and also lists alternatives.

\section{SYSTEM DESCRIPTION}

The ILC Beam Delivery System (BDS) is responsible for transporting the $\mathrm{e}^{+} / \mathrm{e}^{-}$beams from the exit of the high energy linacs, focusing them to the sizes required to meet the ILC luminosity goals $\left(\sigma_{x}^{*}=639 \mathrm{~nm}, \sigma_{y}^{*}=5.7 \mathrm{~nm}\right.$ in the nominal parameters), bringing them into collision, and transporting the spent beams to the main beam dumps. In addition, the BDS must measure the linac beam and match it into the final focus (FF); protect the beamline and detector against mis-steered beams from the main linacs; remove any large amplitude particles (beam-halo) from the linac to minimize background in the detectors; measure and monitor the key physics parameters such as energy and polarization before and after the collisions. The BDS must provide sufficient instrumentation, diagnostics and feedback systems to achieve these goals.

The main subsystems of the beam delivery starting from the exit of the main linacs are the diagnostics region, fast extraction and tuneup beamline, betatron and energy collimation, final focus, interaction region (IR) and extraction line. The layout of the beam delivery system is shown in Figs. 1 and 2. The BDS is designed for $500 \mathrm{GeV} \mathrm{CM}$ but

\footnotetext{
* Work supported in part by Department of Energy contract DE-AC02$76 \mathrm{SF} 00515$.
}

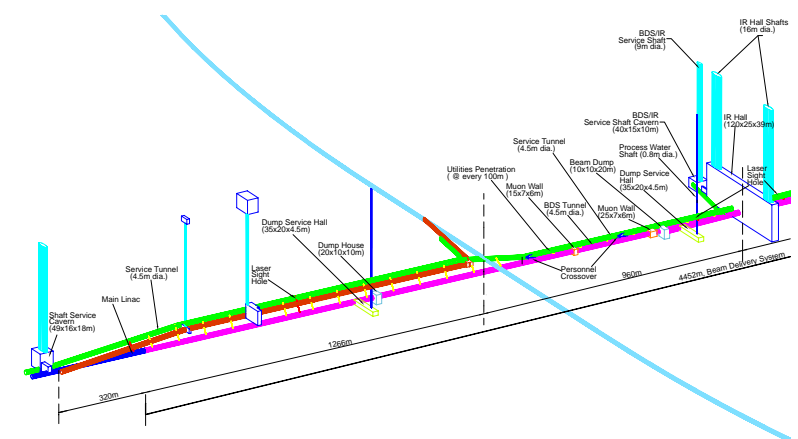

Figure 1: BDS layout, beam and service tunnels (shown in magenta and green), shafts, experimental hall.

can be upgraded to $1 \mathrm{TeV}$ with additional magnets.

There is a single collision point with a 14 mrad crossing angle. The beam delivery systems are in line with the linacs and the linacs are also oriented at a $14 \mathrm{mrad}$ angle. Two detectors in a common IR hall alternately occupy the single collision point, in a so-called "push-pull" configuration. The detectors are pre-assembled on the surface and then lowered into the IR hall in large subsections once the hall is ready for occupancy.

The initial part of the BDS is responsible for measuring and correcting the properties of the beam before it enters the collimation and FF. In addition, errant beams must be detected here and safely extracted in order to protect the downstream systems. Starting at the exit of the main linac, the system includes the machine protection system (MPS) collimation, skew correction section, emittance diagnostic section, polarimeter with energy diagnostics, fast extraction/tuning system and beta matching section.

At the exit of the main linac is a short $90^{\circ}$ FODO lattice, composed of large bore quadrupoles, which contains a set of sacrificial collimators of decreasing aperture. This section also contains kickers and BPMs for inter- and intratrain trajectory feedback. 

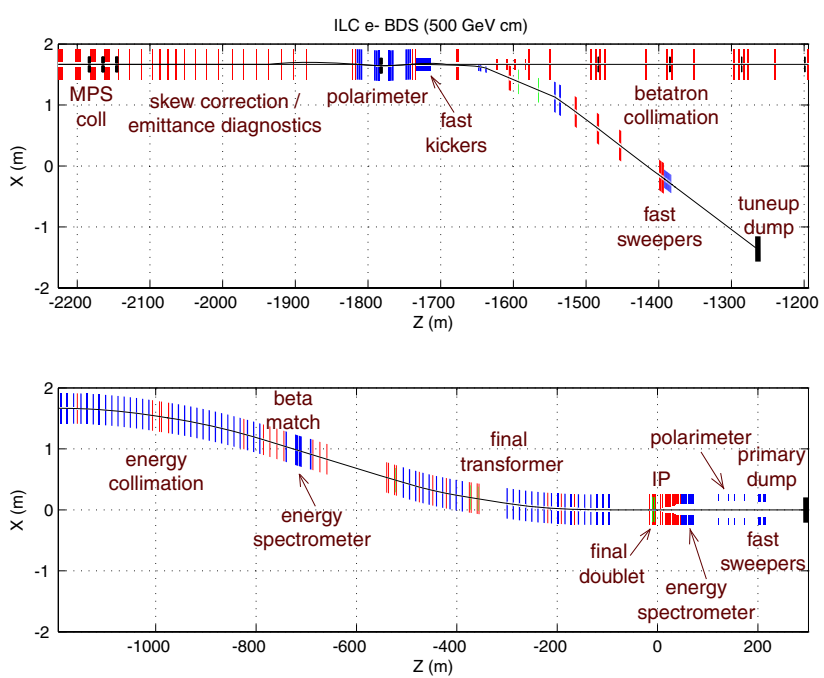

Figure 2: BDS layout showing functional subsystems, starting from the linac exit.

The skew correction section contains 4 skew quadrupoles which provide complete and orthogonal independent control of the 4 betatron coupling parameters. This scheme allows correction of any arbitrary linearly coupled beam.

The emittance diagnostic section contains 4 laser wires which are capable of measuring horizontal and vertical RMS beam sizes down to $1 \mu \mathrm{m}$. The wire scanners are separated by $45^{\circ}$ in betatron phase to allow a complete measurement of $2 \mathrm{D}$ transverse phase space and determination of the projected horizontal and vertical emittances.

Following the emittance diagnostic section is a magnetic chicane which is used for both Compton polarimetry and beam energy diagnostics. At the center of the chicane is the Compton IP, a cavity BPM for measuring relative energy changes, and a sacrificial MPS energy collimator which defines the energy acceptance of the tuneup extraction line.

The pulsed extraction system is used to extract beams in the event of an intra-train MPS fault. It is also used when beams are not desired in the collimation, final focus, or IR areas, for example during commissioning of the linacs. The extraction system includes both fast kickers which can rise to full strength in the $300 \mathrm{~ns}$ between bunches, and pulsed bends which can rise to full strength in the $200 \mathrm{~ms}$ between trains. These are followed by a transfer line with $\pm 10 \%$ momentum acceptance which transports the beam to a fullbeam-power water-filled dump. By using the nearby and upstream BPMs in the polarimeter chicane and emittance sections, it is possible to limit the number of errant bunches which pass into the collimation system to 1-2.

Particles in the beam halo produce backgrounds in the detector and must be removed in the BDS collimation system. The collimation system has a betatron collimation section followed by energy collimators. The downstream energy collimators help to remove the degraded energy particles originating from the betatron collimation section but not absorbed there. The betatron collimation system has two spoiler/absorber x/y pairs located at high beta points, providing single-stage collimation at each of the final doublet (FD) and IP betatron phases. The energy collimation section has a single spoiler located at the central high dispersion point $(1530 \mu \mathrm{m} / \%)$. All spoilers and absorbers have adjustable gaps. Protection collimators (PC) are located throughout to provide local protection of components and additional absorption of scattered halo particles.

The spoilers are 0.5 to $1 \mathrm{X}_{0}$ (radiation length) thick, the absorbers are $30 \mathrm{X}_{0}$, and the protection collimators are $45 \mathrm{X}_{0}$. The betatron spoilers as well as the energy spoiler are "survivable" - they can withstand a hit of two errant bunches of $250 \mathrm{GeV} /$ beam, matching the emergency extraction design goal. With $500 \mathrm{GeV}$ beam, they would survive only one bunch, and would therefore require more effective MPS or the use of a pre-radiator scheme.

Electromagnetic showers created by primary beam particles in the collimators produce penetrating muons which can easily reach the collider hall. The muon flux through the detector is reduced by a $5 \mathrm{~m}$ long magnetized iron shield located $330 \mathrm{~m}$ upstream of the collision point which fills the cross-sectional area of the tunnel. The shield also provides radiation protection for the IR hall during access periods when beam is present in the linac and tuneup line.

With the $5 \mathrm{~m}$ wall and with $1-2 \times 10^{-5}$ of the beam collimated, the number of muons reaching the collider hall would be a few muons per 150 bunches. To cope with uncertainty in halo predictions, the BDS includes caverns large enough to increase the muon shield from $5 \mathrm{~m}$ to $18 \mathrm{~m}$ and to add an additional $9 \mathrm{~m}$ shield downstream. Filling all of these caverns with magnetized muon shields would increase the muon suppression capacity of the system to $1 \times 10^{-3}$ of the beam. The primary beam spoilers and absorbers are water cooled and capable of absorbing $1 \times 10^{-3}$ of the beam continuously.

The final focus system demagnifies the beam to the required size and provides local chromaticity correction [1] using sextupoles next to the final doublets. The FF includes space for additional bend magnets which are necessary at energies above $500 \mathrm{GeV} \mathrm{CM}$. The final focus includes two superconducting octupole doublets which use nonlinear focusing to reduce the amplitudes of beam halo particles while leaving the beam core untouched [2]. This "tail-folding" would permit larger collimation amplitudes, which in turn would reduce the amount of beam power intercepted and the unwanted wakefields.

The ILC final focus uses independently adjustable compact superconducting magnets for the incoming FD and extraction beam lines [3]. The BNL direct wind technology is used to produce closely spaced coil layers of superconducting multi-strand cable. Cooling is provided by superfluid helium at $2 \mathrm{~K}$. The technology has been demonstrated by a series of short prototype multi-pole coils. For the quadrupoles closest to the IP the magnetic cross talk between the two beam apertures is controlled by using actively shielded coil configurations and by use of local correction coils. The IR optics also include an anti-solenoid 
and a large aperture Detector Integrated Dipole (DID).

Crab cavities rotate the bunches so they collide head on. Two $3.9 \mathrm{GHz}$ SC 9-cell cavities in a 2-3 m long cryomodule are located $13.4 \mathrm{~m}$ from the IP and operate at $5 \mathrm{MV} / \mathrm{m}$ peak deflecting gradient [4]. The cavities are based on the

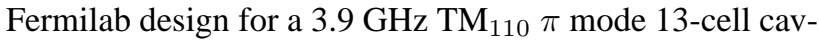
ity. The uncorrelated phase jitter between the positron and electron cavities must be controlled to $61 \mathrm{fsec}$. A proof-ofprinciple test of a 7 cell $1.5 \mathrm{GHz}$ cavity at the JLab ERL facility [5] has achieved a 37 fsec level of control, demonstrating feasibility. The higher- and lower-order modes of the cavity must be damped to limit unwanted vertical deflections at the IP, as must the vertical polarization of the main deflecting mode.

The extraction line transports the beams from the IP to the dump with minimal beam losses, while providing dedicated optics for beam diagnostics. The extraction line can transport particles with momentum offsets of up to $60 \%$. There is no net bending in the extraction line, which allows the charged particle dump to also act as a dump for beamstrahlung photons with angles of $\pm 0.75 \mathrm{mrad}$.

Maintaining the overall stability of the BDS is an essential prerequisite to producing luminosity. Care must be taken to minimize thermal and mechanical disturbances, by stabilizing the air temperature, cooling water, and by limiting high frequency vibrations due to local equipment. Beam-based feedback loops which are used to maintain the size and position of the beam at the IP include train-by-train feedbacks, operating at the $5 \mathrm{~Hz}$ repetition rate of the ILC, and intra-train feedbacks, which can apply a correction to the beam between bunches of a single train.

Absolute beam energy measurements at better than $200 \mathrm{ppm}$ are achieved with two independent and complementary spectrometers for each beam. Upstream from the IP - a BPM based spectrometer, and downstream from the IP - a synchrotron radiation spectrometer. The ILC luminosity can be measured with a precision of $10^{-3}$ or better by measuring the Bhabha rate with the LumiCal detector located in front of the FD. Precise polarimetry with $0.25 \%$ accuracy is achieved with Compton polarimeters located both upstream and downstream of the IP.

The BDS contains two tune-up dumps and two main beam dumps. These four dumps are all designed for a peak beam power at nominal parameters of $17 \mathrm{MW}$ at $500 \mathrm{GeV}$ per beam. These dumps consist of $1.5 \mathrm{~m}$ diameter cylindrical stainless steel high pressure (10 bar) water vessels with a $30 \mathrm{~cm}$ diameter $1 \mathrm{~mm}$ thick Ti window; and also include their shielding and associated water systems. Each dump incorporates a beam sweeping magnet system to move the charged beam spot in a circular arc of $3 \mathrm{~cm}$ radius during the passage of the $1 \mathrm{~ms}$ long bunch train.

Almost all BDS magnets are conventional. The bend magnets in the FF have fields of less than $0.5 \mathrm{kG}$ to minimize synchrotron radiation. The main technical issue with the BDS magnets is their positional stability. All the incoming beamline quadrupoles and sextupoles sit on 5 degree of freedom magnet movers with a $50 \mathrm{~nm}$ step.
The design of the vacuum system is governed by resistive and geometric wakes [6] and beam-gas scattering. The latter requires the vacuum to be about $1 \mathrm{nTorr}$ within $200 \mathrm{~m}$ of the IP and 10-50 nTorr further away from the IP.

To facilitate a rapid, "push-pull" style exchange of detectors at a shared IP, the superconducting final focus magnets are arranged into two groups so that they can be housed in two separate cryostats, with only warm components and vacuum valves placed in between, in the breakpoint. A necessary condition for efficient push-pull operation is to avoid disconnecting any of the systems for the detectors during the exchange. One possible solution is to equip each detector with an adjacent service platform which moves together with the detector. The platforms would house the cryogenic systems, high current power supplies for solenoids and FD, and detector electronics. All the connections between the platform and detector would be fixed and not disconnected during the exchange. The movable detector service platform would have flexible connections to fixed services (including high voltage $\mathrm{AC}$, room temperature high pressure He supply and return, data $\mathrm{I} / \mathrm{O}$, etc.), that do not need to be disconnected during the exchange.

The above outline describes the baseline design of the BDS. For several subsystems there are alternative designs which are being pursued. In particular, the design of headon and $2 \mathrm{mrad}$ crossing angle configurations is continues $[7,8]$ focusing on designing the most economical system (without downstream diagnostics, while alternative ideas of polarimetry at IP and spectrometry just after IP are being discussed). Other alternatives include consumable instead of survivable spoilers; distributed muon collars instead of localized muon walls; normal conducting or lower frequency (1.3 or $2.6 \mathrm{GHz}) \mathrm{SC}$ crab cavity; an additional intra-train feedback loop at the entrance to BDS; beam dump based on a km long pipe filled with noble gas; use of a Fabry-Perot cavity for the laser at the Compton IP at the polarimeter; use of adjustable permanent magnets for the final doublet; use of Compton backscattering for precise beam energy measurement; modification of downstream polarimetry chicane to increase spectral coverage of Compton electrons and ease use of a gamma calorimeter.

\section{REFERENCES}

[1] P. Raimondi and A. Seryi, "A novel final focus design for future linear colliders," Phys. Rev. Lett. 86, 3779 (2001).

[2] R. Brinkmann, P. Raimondi, A. Seryi, "Halo reduction by means of nonlinear optical elements in the NLC final focus system," SLAC-PUB-8896, PAC01 (2001).

[3] B. Parker et al, The Superconducting Magnets of the ILC Beam Delivery System, in these Proceedings.

[4] L. Xiao et al, Crab Cavity Design for the ILC; G. Burt et al, Couplers; A. Dexter et al, RF control, in these Proceedings.

[5] M. Liepe et al, Pushing the limits: RF field control at high loaded Q, PAC2005.

[6] K. Bane et al, Wakefield Effects in the Beam Delivery System of the ILC, in these Proceedings.

[7] O. Napoly et al, Technical Challenges for Head-On Collisions and Extraction at the ILC, in these Proceedings.

[8] R. Appleby et al, Improved 2mrad extraction line layout for the International Linear Collider, in these Proceedings. 